\title{
INVESTIGATING THE EFFECTS OF RIVER DISCHARGES ON SUBMERGED AQUATIC VEGETATION USING UAV IMAGES AND GIS TECHNIQUES
}

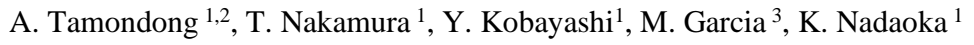 \\ ${ }^{1}$ Department of Transdisciplinary Science and Engineering, Tokyo Institute of Technology, Tokyo, Japan \\ ${ }^{2}$ Department of Geodetic Engineering, University of the Philippines, Quezon City, Philippines - amtamondong@ up.edu.ph \\ ${ }^{3}$ Marine Science Institute, University of the Philippines, Quezon City, Philippines
}

\begin{abstract}
Youth Forum
KEY WORDS: SAV, UAV, DJI Phantom, seagrass, seaweeds, spatial interpolation, water quality
\end{abstract}

\begin{abstract}
:
One of the major factors controlling the distribution and abundance of marine submerged aquatic vegetation (SAV) is light availability. Reduced water clarity due to sediment loading from rivers greatly affects the health and coverage of seagrasses and seaweeds. Monitoring SAV using unmanned aerial vehicles (UAV) has been getting attention because of its cost-effectiveness and ease of use. In this research, a low-cost UAV was utilized to assess the impacts of river discharges on SAV in Busuanga Island, Philippines. Linear regression was performed to determine the effectivity and accuracy of UAV-based percent cover estimation compared to established field survey methods of monitoring SAV. Water quality was estimated in the study area by performing spatial interpolation methods of in situ measurement of turbidity, chlorophyll, temperature, salinity, and dissolved oxygen using a multi-parameter water quality sensor. Current velocity and tidal fluctuations were monitored using bottom-mounted sensors deployed near the river mouth and in seagrass and seaweed areas with relatively good water clarities. Four stations were surveyed using automated UAV missions which were flown simultaneously with field observations. Each station surveyed has varying distances from the river mouth. Results from the classification of the UAV data and field survey show that SAV is more abundant as the distance from the river mouth increases and the turbidity decreases. Classification overall accuracies of UAV orthophotos ranging from $87.91-93.41 \%$ were achieved using Maximum Likelihood (ML) Classification. Comparison of field-based and UAV-based survey of percent cover of seagrasses show an overestimation of 1.75 times from the UAV compared to field observations.
\end{abstract}

\section{INTRODUCTION}

Submerged aquatic vegetation (SAV) plays an important role in the coastal ecosystem. They support a diverse group of fauna and flora as they serve as shelter and breeding grounds to several marine species and also provide for their food. (Ackleson and Klemas, 1987; Orth and Moore, 1984). Aside from that, they also serve as bottom sediment stabilizers and act as buffers of water flow (Komatsu et al., 2002). Seagrasses, considered as SAV, are marine flowering plants with roots, while seaweeds, also known as macroalgae, are freely floating plants (Kolanjinathan et al., 2014). Unfortunately, SAV is among the most neglected ecosystems. In the Philippines, they are less protected by environmental laws compared to other coastal ecosystems such as mangroves and coral reefs which lead to the loss of these important habitats in the country.

There has been an increasing loss of SAV cover throughout the world, most of them undocumented (Green and Short, 2004). Several factors contribute to the decline of SAV coverage which includes natural and anthropogenic factors. The changing climate and environment will only aggravate the decline of these important blue carbon ecosystems. The monitoring of these habitats is essential and needed for proper coastal planning and management. However, field-based monitoring methods are costly and time-consuming. Therefore, a need for a cost-effective and easy method of monitoring SAV arises.

The use of remote sensing as a means to monitor SAV (Ackleson and Klemas, 1987) has been getting popular because of advancements in satellite technology (Yuan and Zhang, 2008) and development of low-cost unmanned aerial vehicles (UAV) (Flynn and Chapra, 2014). However, monitoring SAV using optical remote sensing usually requires the study area to have clear waters. Optical remote sensing of the marine environment is restricted by the exponential attenuation of light radiations in the water (Smara et al., 1998). Tropical coastal areas, like the Philippines, are usually affected by terrestrial runoff from rivers which can significantly increase water turbidity. Turbid waters may cause misclassifications and low accuracies in mapping SAV using remotely sensed data (McCarthy and Sabol, 2000).

River discharges hinder the use of optical remote sensing for SAV mapping and they also affect the distribution and abundance of the coastal ecosystem. Suspended sediments from the river cause turbidity which limits the light penetrating the water column. Light availability is an important factor for SAV to thrive underwater (Koch, 2001). River runoff may also cause nutrient enrichment which may increase the presence of phytoplankton. Consequences due to this phenomenon may include increased concentrations of chlorophyll-a and depletion of oxygen which may result in shifts in species composition, increased growth of epiphytic algae, and loss of SAV (Devlin et al., 2011). To be able to manage the coastal resources properly and prevent the loss of SAV, it is important to monitor their condition and the surrounding environment using cost-efficient and less time-consuming methods.

The main objective of this research is to investigate the effects of river discharges on the distribution and abundance of SAV in the study area. This is carried out by processing data from ocean monitoring sensors, UAV images, and field observations using remote sensing and geographic information system (GIS) methods. It also aims to determine the applicability of using UAV for monitoring SAV habitats. 


\section{MATERIALS AND METHODS}

\subsection{Study Area}

The study area is in Busuanga Island which is situated in the Northern part of Palawan province in the Philippines. It is a $3^{\text {rd }}$ class municipality with an estimated population of 22,046 in the year 2015 (Philippine Statistic Authority, 2015). The coastal ecosystem in Busuanga is relatively pristine compared to other parts of the country which are degraded due to human-induced disturbances, tourism, bad aquaculture practices, direct mechanical damages, release of toxic compounds into the coastal waters, etc. Mangroves, seagrass, seaweeds, and coral reefs are abundant and thriving in Busuanga. There are several rivers present on the island, however, only one river was selected as a study site (Figure 1). This river was nearest an established marine protected area which means human-induced disturbances in the area are limited. Moreover, the site is selected because of the presence of SAV in areas near and far from the river mouth.

Four stations (A, B, C, and D) were selected in the study area. As shown in Figure1, each station has varying distances from the river mouth, wherein, Station A is the closest ( $\sim 300$ meters) while Station D ( 5000 meters) is the farthest.

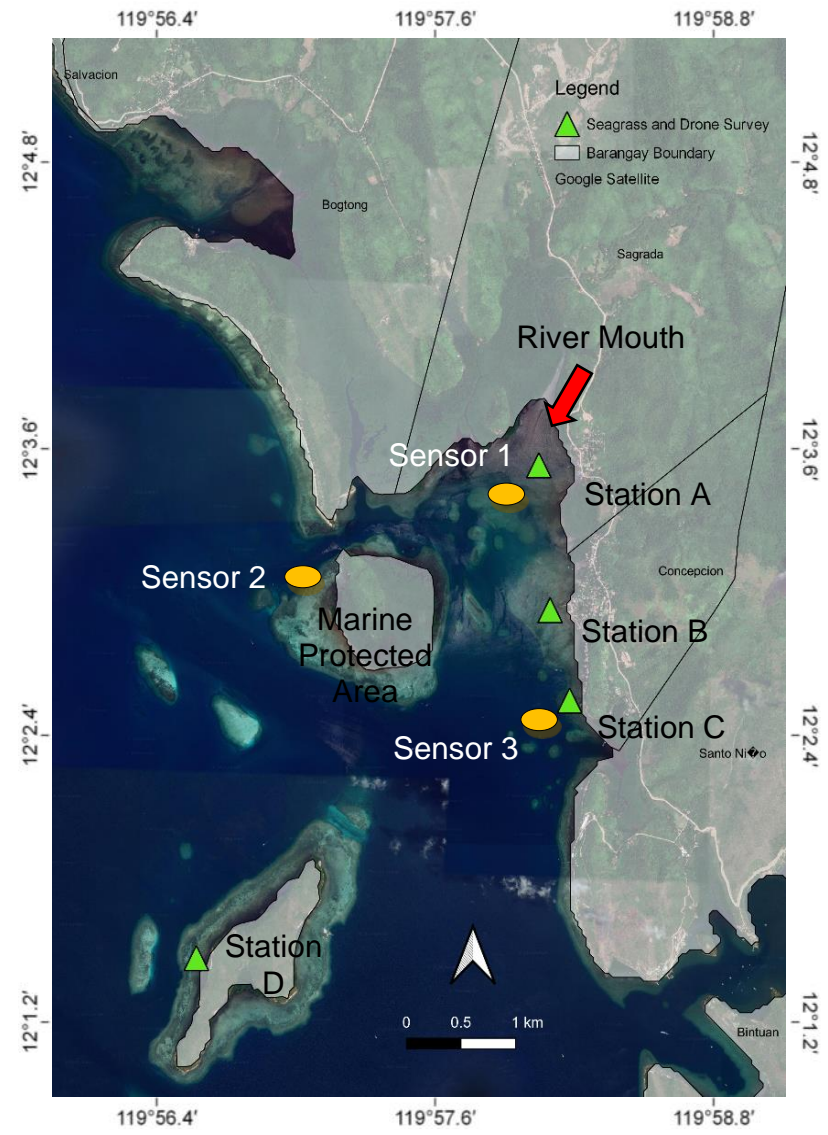

Figure 1. Location of SAV surveys (A, B, C, D), UAV surveys (A, B, C, D) and sensor deployments $(1,2,3)$

\subsection{Field Data Gathering}

Before going to the field, a Sentinel-2A multispectral satellite image of the study area was processed and classified. IsoDATA, an unsupervised classification method, was performed to map and determine the location of SAV in the study area. The
Sentinel-2A image was also used to determine the relative depth of the area which was utilized in the planning stage of the field survey. The field survey was conducted last September 22-29, 2019 in Busuanga, Palawan. It comprised of four major surveys: sensor deployment, water quality survey, UAV flight missions, and SAV field survey.

At the start of the field survey, water quality assessment was conducted using an AAQ Rinko Profiler in 18 different locations within the study site. The AAQ Rinko is a multi-parameter sensor that can measure depth, chlorophyll, turbidity, dissolved oxygen, $\mathrm{pH}$, temperature, and salinity. A spatial interpolation method was performed later on to estimate the water quality distribution in the study area. Reconnaissance of possible locations of sensor deployment was also assessed simultaneously with the gathering of water quality parameters.

The ocean monitoring sensors were deployed in three different locations (Figure 1) using a bottom-mounted set-up (Figure 2). Various sensors to assess the current velocity, tidal fluctuations, and turbidity in the study area were included. To measure current speed and direction, an Infinity Electro-Magnetic (EM) sensor was deployed while a compact CLW was used to measure chlorophyll and turbidity values. Both sensors are made by JFE Advantech. Additionally, to monitor the water level and temperature, a HOBO water level logger was added to the set-up. Figure 2 shows a photo of the set-up with the sensors deployed near the river mouth (Sensor 1/Station A). To make sure that the presence or absence of river discharges will be captured by the sensors, set-ups were made at shallow depths (1-3 meters at low tide) with minimal obstructions of water flow in their surroundings. It was also made sure that the sensors will not breach the water surface during the lowest tide of the day.

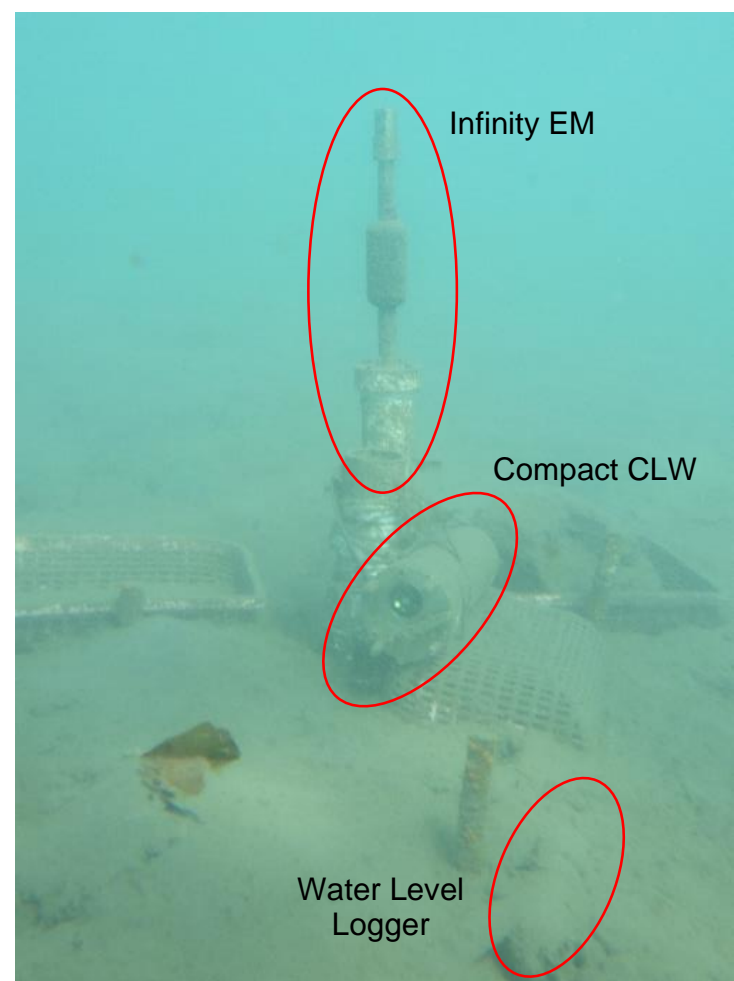

Figure 2. Photo of the deployed bottom-mounted set-up near the river mouth 
The next task was to conduct UAV flight missions simultaneously with field observations of SAV using the Seagrass-Watch protocol (Seagrass-Watch, 2010). For the UAV survey, a DJI Phantom 4 Pro V2 drone was flown autonomously in the four stations. The camera sensor mounted on the drone is only capable of capturing the red, green, and blue (RGB) wavelength ranges of the electromagnetic spectrum which is one of the limitations of this study. During the survey, a 30-meter flying height was kept constant for all the stations. However, additional 50-meter altitude flights were also conducted as backup data. This was proven useful as the most turbid area (Station A) was difficult to process because of the lack of visible tie points in the UAV images. The combination of images from both 30-meter and 50-meter flights was successfully processed and an orthophoto was generated. The camera angle was kept at nadir for all the flight missions. Also, all flight missions were conducted during the low tides wherein the effects of depth and turbidity are minimal. Another factor to consider when doing flight missions is to minimize the presence of sun glint and whitewash due to waves on the images. Fortunately, sun glint and whitewash were minimal in the images gathered for this research. Furthermore, it is also easier to do the SAV survey during low tide

For the SAV survey, the methods of Seagrass-Watch for the Philippines were followed. In this protocol, three 50-meter parallel transects, 25 meters apart, were laid out per station. Ground controls points using colorful buoys with weights were established at the end of the transects. This way, they will be easily identifiable in the drone images. This enabled the correct identification of the location of the transects for analysis and accuracy assessment, wherein usually, handheld GPS was used to mark the position of the transects. A handheld GPS, however, may causes blunders in the validation of classifications due to its low positional accuracy. Highly accurate positioning equipment such as Differential GPS is more recommended to use than handheld GPS. However, they are too expensive and are more prone to damages due to seawater exposure. Therefore, using buoys to mark the transects is a cheaper, easier, and more accurate alternative to handheld GPS. For every transect, a $50 \mathrm{~cm}$ by $50 \mathrm{~cm}$ quadrat was laid out every 5 meters to monitor the habitats present in the area. Field observations were written on waterproof slates and photos of each quadrat were taken. Data gathered from each quadrat were seagrass percent cover, species, canopy height, and epiphyte cover. Seaweed percent cover was also encoded. All stations of SAV surveys were observed to be less than two meters in depth.

\subsection{Data Processing}

The water quality measurements from the AAQ Rinko Profiler were interpolated using the Spline Interpolation with Barrier tool in (CArcMap. It approximates values using a mathematical function that reduces overall surface curvature which produces a smooth surface that passes precisely through the input points. This interpolation method is appropriate for producing gently varying surfaces such as pollution concentrations (ESRI, 2016). In concept, the spline curves a plane that passes through the input points while lessening the overall curvature of the surface. (Briggs, 1974; ESRI, 2016). The results of the interpolation and the location of the AAQ measurements are shown in Figure 3. Using this map, relative turbidity between the stations of SAV and UAV survey can be determined.

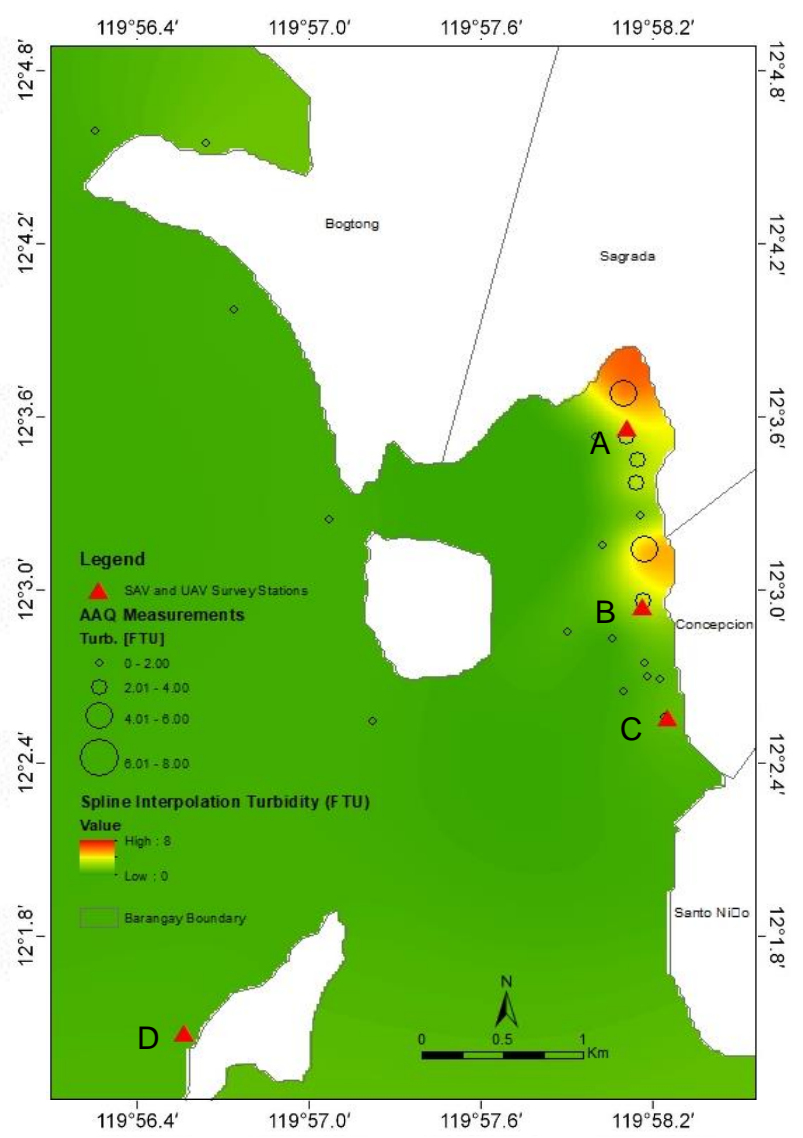

Figure 3. Map of turbidity measurements using AAQ

The UAV images gathered were processed using (CAgisoft Photoscan to create orthophotos. The settings used were high accuracy for the alignment of photos and medium accuracy for building the dense cloud and mesh. Table 1 shows the summary of the parameters of the UAV surveys such as flying height, no. of photos aligned, and spatial resolution of the output orthophoto. In Station A, which was nearest the river mouth, a combination of images from the 30-m and 50-m flights were used for image matching/determination of image tie points for photo alignment. Images from the $30-\mathrm{m}$ flights are insufficient due to lack of visible tie points in this highly turbid area nearest the river mouth. Combining the two sets of flying heights, a total of 520 photos were aligned successfully. The drawback was a lower spatial resolution orthophoto compared to the other stations (Table 1.) The spatial resolution of the generated orthophotos was suitable for distinguishing seagrasses, seaweeds, and sand using visual inspection. High spatial resolution data was successfully produced with minimal costs and less time in the field. However, using UAV also has disadvantages. The reduced time spent in the field was replaced by long processing time in the computer laboratory and the lower cost was offset by expensive computer requirements such as large storage capability and fast processing power.

\begin{tabular}{|c|c|c|c|}
\hline Station & $\begin{array}{c}\text { Flying } \\
\text { Height }\end{array}$ & $\begin{array}{c}\text { No. of photos } \\
\text { aligned }\end{array}$ & $\begin{array}{c}\text { Spatial Resolution } \\
\text { of Orthophoto }\end{array}$ \\
\hline $\mathrm{A}$ & $30 \mathrm{~m}+50 \mathrm{~m}$ & 520 & $1.27 \mathrm{~cm}$ \\
\hline $\mathrm{B}$ & $30 \mathrm{~m}$ & 439 & $0.88 \mathrm{~cm}$ \\
\hline $\mathrm{C}$ & $30 \mathrm{~m}$ & 307 & $0.82 \mathrm{~cm}$ \\
\hline $\mathrm{D}$ & $30 \mathrm{~m}$ & 456 & $0.79 \mathrm{~cm}$ \\
\hline
\end{tabular}

Table 1. Summary of UAV Processing Information 
The resulting orthophotos, shown in Figure 4, were classified using Maximum Likelihood (ML) Classification, one of the most widely used parametric algorithm (Jensen, 2014). ML uses the training data to estimate the mean and variances of the classes which are then utilized to compute for probabilities (Campbell and Wynne, 2011). This is advantageous if you have good training datasets, which is the case when using UAV data, because of the easily identifiable objects in high spatial resolution data. Before performing the classification, land features were masked out as well as floating objects such as boats because they are not of interest in this study. Masking unnecessary objects will also lessen the number of classes for classification and reduce possible errors. Then, a combination of image interpretation and field observation data was utilized to generate training datasets for classification. Accuracies of the classifications were determined using confusion matrices.
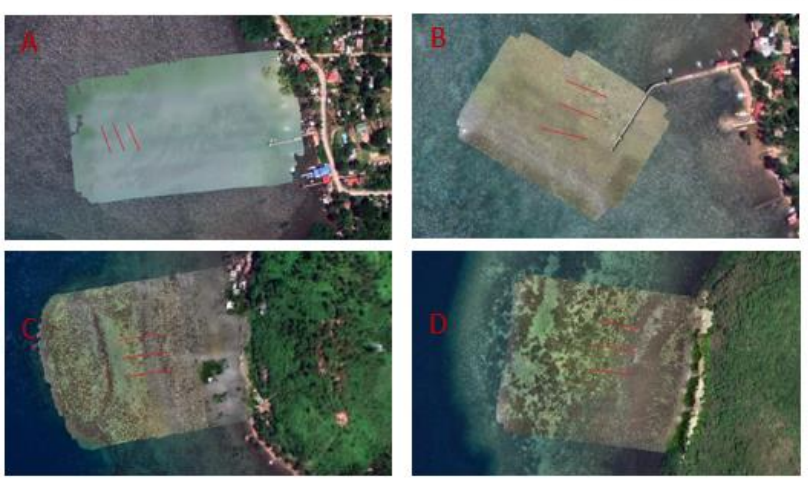

Figure 4. Processed UAV orthophotos and SAV transect plots overlaid in (CGoogle Earth images

To further assess the output of the classified images, a comparison of UAV-based and field-based seagrass percent cover estimation was performed and analyzed using linear regression. This was done by digitizing the quadrats on the orthophotos and then calculating the zonal statistics of the classified seagrasses per quadrat. Figure 5 shows the steps to calculate the percent cover of seagrass from UAV classified images. The relationship between the UAV-based seagrass quadrat statistics and field observations was determined using linear regression.

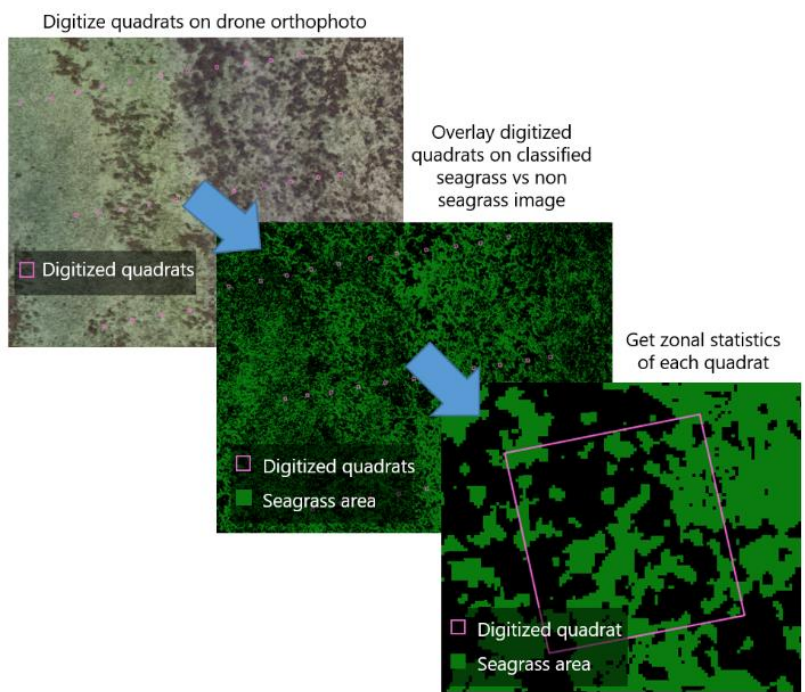

Figure 5. Process of calculating the percent cover of seagrass from UAV classified images

\section{RESULTS AND DISCUSSION}

\subsection{Sensor Deployment and Water Quality Survey}

Based on the data from the deployed sensors and water quality survey, Station A, which is nearest the river mouth, has the highest turbidity while Stations C and D, farthest from the river mouth, have low turbidity values (Figure 3 ). The turbidity readings in Station B fall in the middle range of Stations A and C. Additionally, salinity values are lowest in Station A, as shown in Figure 6. This indicates a mixing of river and seawater in the area. Stations $\mathrm{C}$ and $\mathrm{D}$ have the highest salinity values signifying minimal to zero influence from the river discharges. Station B, on the other hand, has high salinity readings (Figure 6) but also high turbidity (Figure 3 ) which indicates that there other possible sources of suspended sediments in the area which are not covered by the scope of this study.

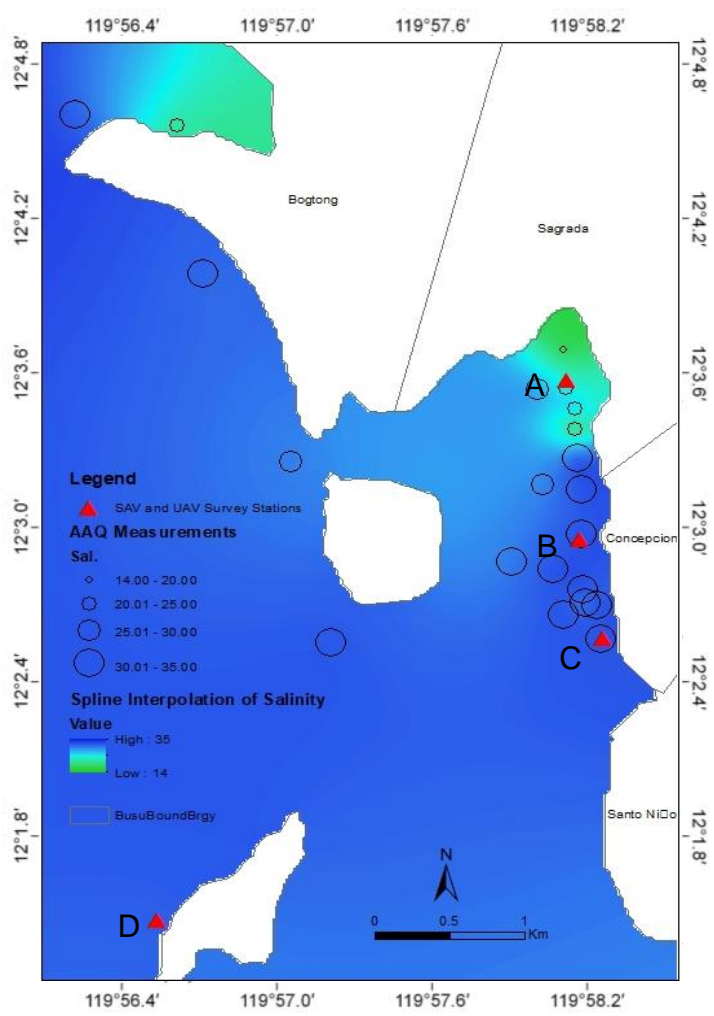

Figure 6. Map of salinity measurements using AAQ

Results from the water level logger (Figure 7) deployed near Station A show the change in water pressure which indicates a diurnal tide from September 24-25, 2019 while a mixed semidiurnal tide happened from September 26-29, 2019. This plot was already corrected for atmospheric pressure by subtracting values from a water level logger left at the surface during the survey. UAV flight missions were conducted during the low tide of the day, which usually occurs in the afternoon as indicated in the water level logger data.

Furthermore, the Infinity EM and compact CLW data indicate that water temperature and turbidity increase when water is coming from the river. The direction of the current recorded by the Infinity EM indicates that the water came from the river, going to the direction of Station A, during periods where high turbidity was logged by the compact CLW. When compared to the data of absolute water pressure, it can be concluded that high turbidity occurs during ebb tide. On the other hand, when the tide is high, low turbidity and temperature values were observed. This 
means that the high turbidity values in the study area are due to river discharges. The SAV station A is located less than 100 meters away from the sensor set-up.

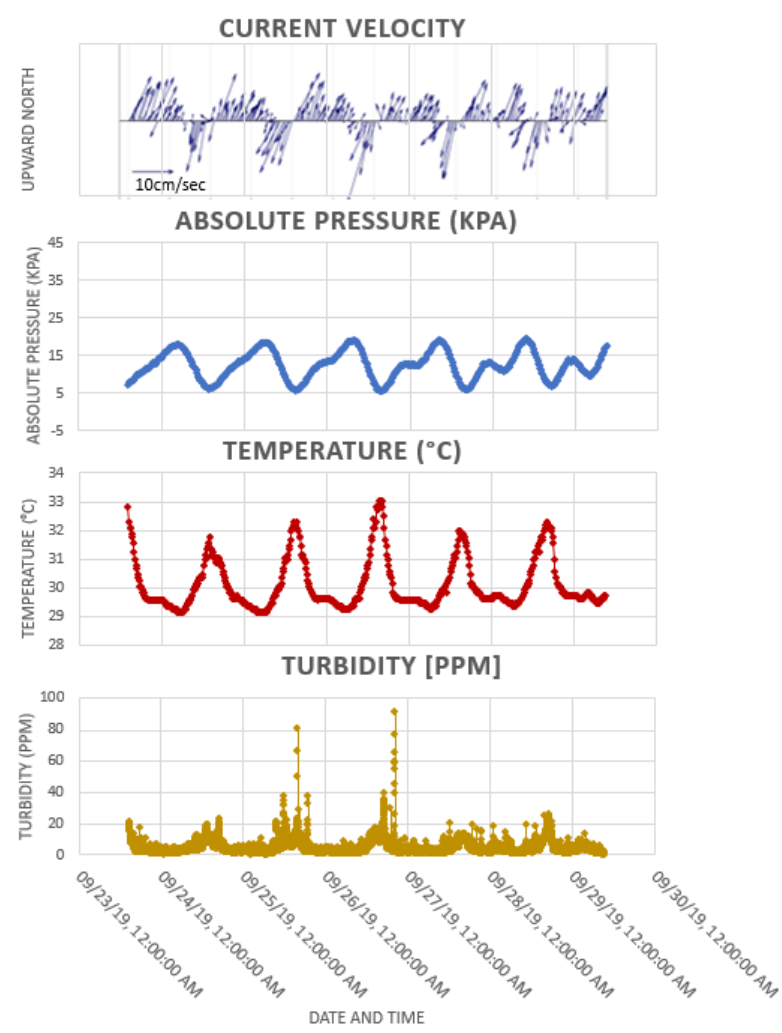

Figure 7. Ocean monitoring sensor readings near the river mouth

\subsection{SAV Survey}

Based on field observations, Enhalus acoroides ( $E a)$ is the only species of seagrass present in Station A. These species are the most resilient to turbid water and can tolerate lower salinity concentrations. On the other hand, only two species were found in Station B, Ea, and Cymodocea serrulata (Cs). Ea and Cs were also present in Station C with the addition of Thalassia hempricii $(T h)$. There were also three seagrass species found in Station D: $\mathrm{Ea}$, Th, and Cymodocea rotundata $(\mathrm{Cr})$. This indicates an increase in the number of seagrass species as the distance from the river mouth increases. Averages of the percent cover per station also show an increase of both seagrass and seaweed percent cover as shown in Figure 8. The average epiphyte covers of seagrasses, on the other hand, decreases from Station A to Station D. Based from these results, it can be determined that as the proximity of the station from the river increases, turbidity decreases, while the number of seagrass species increases and the percent cover of seagrass and seaweeds also increases.

\subsection{UAV Survey}

The processed UAV orthophotos were classified using ML Classification and the results are shown in Figure 9. Initially, because of high turbidity, the classification results in Station A was expected to have low accuracy. However, an overall accuracy of $90.16 \%$ was achieved with a kappa coefficient of 0.8036. The reason for this is because, after masking land features, only two classes remain: seagrass and silt/sand. No seaweed was present in Station A.

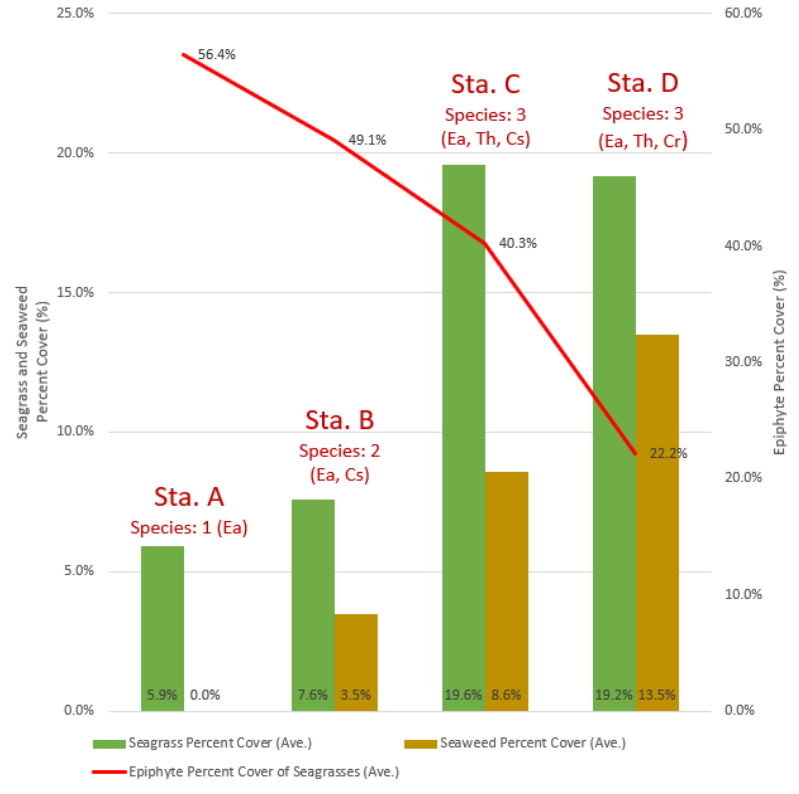

Figure 8. Summary of SAV field survey showing averages of seagrass and seaweed percent covers and average epiphyte cover of seagrasses

On the other hand, in Stations B, C, and D, the presence of seaweeds were observed. The overall accuracies obtained for those three stations were $93.41 \%, 87.91 \%$, and $90.22 \%$ respectively. The relatively low accuracy in Station C was due to changes in cloud cover during the flight mission, which consequently, affected the exposure of the UAV images. Water column correction was not applied because flight missions were conducted during the low tide of the day. This may also be the reason why the accuracy of seagrass classification in Station A was relatively high. During low tide, the leaf blades of $E a$ were already breaching the water surface negating the effects of turbidity which affects the classification accuracy of remotely sensed data. This proves the capability of using low-cost UAV systems for mapping SAV. It is well recommended to do flight missions during low tide, especially in highly turbid waters.
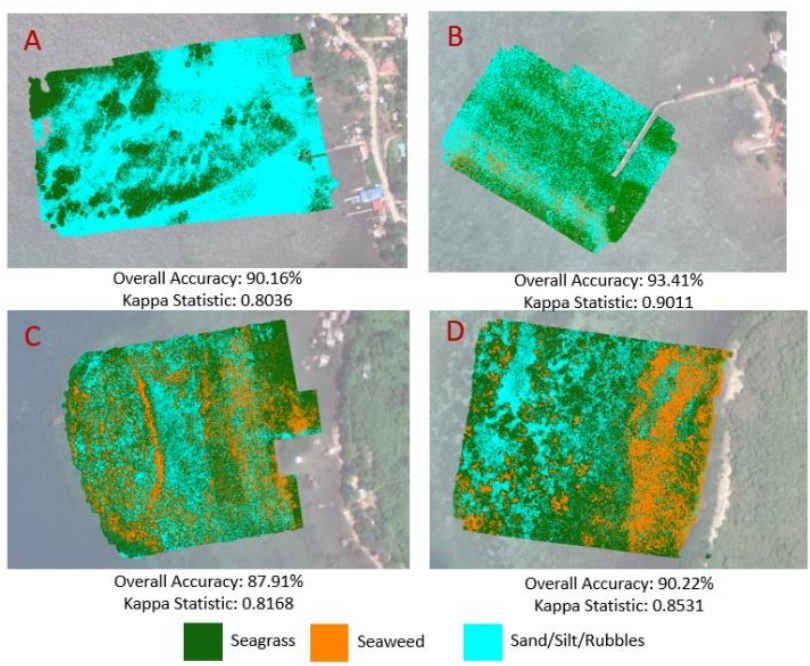

Figure 9. Results of the classification of UAV orthophotos

A study by Taddia et al in 2019 produced reliable results when classifying seagrass from UAV using ML Classification method (Taddia et al., 2019). However, their results indicate that 
performing radiometric calibration before classification will produce better accuracy. This was not tested in this study. Furthermore, a paper by Chayhard et al. in 2018 compared UAV and satellite images for mapping seagrasses (Chayhard et al., 2018). They found out that UAV produced better accuracy than satellite images such as WorldView-2 and GeoEye-1 in classifying long leaves and short leaves type of seagrasses using ML classification.

The UAV-based percent covers of seagrass and seaweed were calculated by dividing the total number of classified pixels of both benthic covers to the total number of pixels in the image. Figure 10 shows the results of this calculation. From this information, it can be concluded that seagrass and seaweed percent cover increase as the distance from the river mouth increases. This result was similar to field observations as shown in Figure 8.

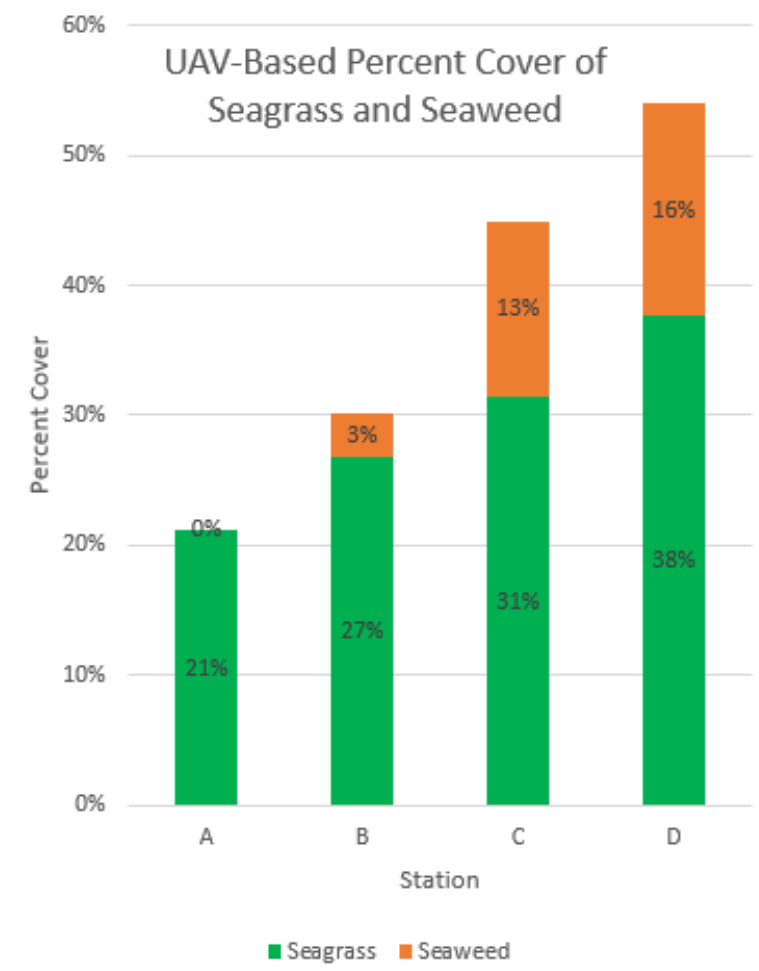

Figure 10. UAV-based percent cover estimation of seagrass and seaweed

The advantage of doing UAV-based measurement is that it is less time consuming, tedious, and biased. From the field survey, one flight will only take approximately 20 minutes, including the setup, to complete, while the field observations took about 2-3 hours, longer in the station with turbid waters. The effort exerted to complete both tasks were huge in disparity. However, there was more information gathered from field observations such as species and epiphyte cover, which is difficult to extract from a low-cost UAV data. To achieve such objective, a multispectral or hyperspectral sensor is needed to separate SAV species using remotely sensed data. On the other hand, the field-based method is more biased in gathering data compared to the UAV-based method. The percent cover extracted from the UAV covers all areas within the flight mission, while the field-based data only captures sampling points within the study area. Nevertheless, results from both methods showed increasing seagrass and seaweed cover as turbidity and distance from the river mouth decrease.
Further comparison of UAV-based and field-based observations was performed using linear regression. Field data from each quadrat were compared to the statistics of the digitized quadrats overlaid on the classified image. A plot of the results for Station $\mathrm{C}$ can be seen in Figure 11 while a summary of the regression statistics for Stations C and D is shown in Table 2. The highest $\mathrm{R}$-squared was 0.7965 which was obtained in Station C. This indicates a relatively good agreement between the UAV-based and field-based estimation of seagrass percent cover.

\begin{tabular}{|l|r|r|}
\hline \multicolumn{1}{|c|}{ Regression Statistics } & \multicolumn{1}{c|}{ Station C } & \multicolumn{1}{c|}{ Station D } \\
\hline R Square & 0.7965 & 0.6762 \\
\hline Adjusted R Square & 0.7652 & 0.6450 \\
\hline Standard Error & 0.2075 & 0.2699 \\
\hline Observations & 33 & 33 \\
\hline
\end{tabular}

Table 2. Summary of regression statistics for seagrass percent cover from drone and field observations

Moreover, the results of linear regression in Station $\mathrm{C}$ indicate that percent cover from the UAV was overestimated 1.7517 times compared to field observations. Incidentally, results from the UAV classification in Station D overestimated the percent cover 1.7515 times compared to field data. When investigating for the probable cause of the overestimation, it was found out that seagrass and seaweed shadows cast on the substrate were also classified at habitat covers which caused the overestimation of percent covers. However, it is also important to note that fieldbased observation of percent cover may be biased. The values during field observation are dependent on the observer and are subjective which can result in over/underestimation. On the other hand, results from the classification of UAV orthophotos are objective because they are calculated from the number of classified pixels.

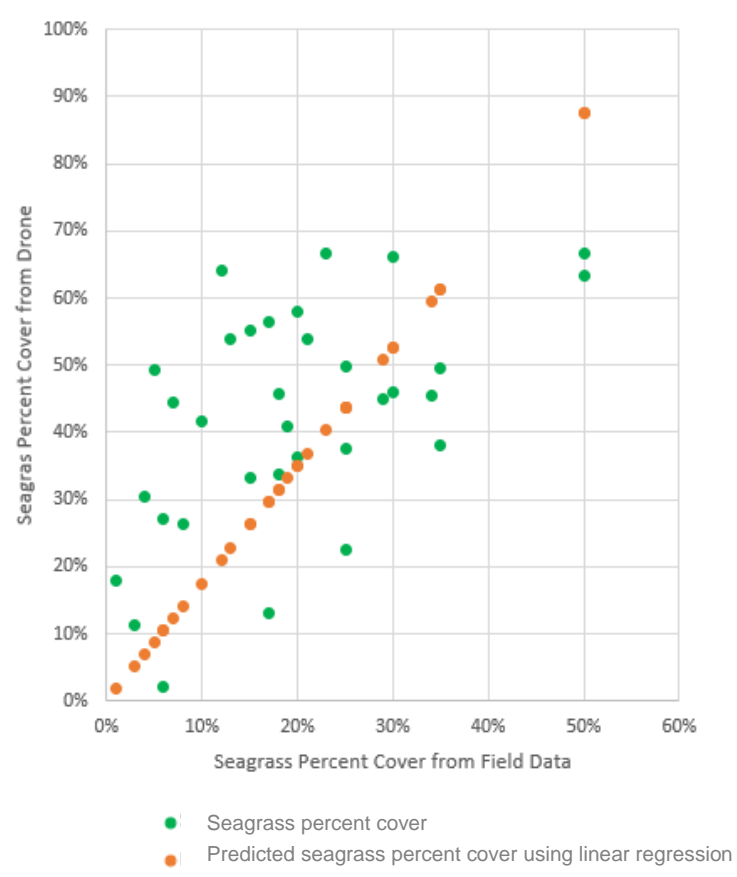

Figure 11. Linear relationship of seagrass percent cover from drone and field data in Station $\mathrm{C}$ 


\section{CONCLUSIONS}

The coastal environment is under threat of loss due to various anthropogenic and natural factors. It is vital to understand the relationships between different marine resources and their surrounding environment which may lead to proper management and protection. SAVs are important marine habitats that are neglected and improperly managed. A cost-efficient and less tedious method of monitoring them was presented in this research. Using Maximum Likelihood (ML) Classification of UAV orthophotos, accuracies ranging from $87.91-93.41 \%$ were obtained in mapping seagrass and seaweed in Busuanga, Palawan. Comparison between percent cover estimation from field observations and classified orthophotos using linear regression indicates a 1.75 multiplier of the values from the UAV versus ground data.

Based on sensor data, classified UAV orthophotos, and SAV field observations, it was found out that water turbidity is highest near the river mouth (Station A). In this station, there are fewer species of seagrass, no presence of seaweed, and low seagrass percent cover. However, as the distance from the river mouth increases, the turbidity decreases while the number of species increases, and the percent cover of seagrass and seaweed also increases.

For future research, it is suggested to investigate the possibility of using high-resolution satellite data as an alternative to UAV images. Freely available satellite images are advancing in terms of spatial and spectral resolution. However, because satellite sensors gather data at a specific time of the day, it is problematic to simultaneously gather field and remotely sensed data during the low tide of the day which is not a problem when using UAV. Moreover, for water quality estimation, numerical modeling may be integrated with the methods presented to improve the study of the effects of river discharges on SAV because temporal analysis may be investigated

\section{ACKNOWLEDGEMENTS}

The authors would like to thank the Comprehensive Assessment and Conservation of Blue Carbon Ecosystems and Their Services in the Coral Triangle (Blue CARES) Project which is under the SATREPS (Science and Technology Research Partnership for Sustainable Development) program funded by JICA-JST (Japan International Cooperation Agency and Japan Science and Technology Agency) for the support of this research. We also appreciate the support given by members of the Nakamura Laboratory at Tokyo Institute of Technology and would also like to thank Dr. Angela Quiros, a postdoc at Hokkaido University, for providing additional field survey data.

\section{REFERENCES}

Ackleson, S.G., Klemas, V., 1987. Remote sensing of submerged aquatic vegetation in lower chesapeake bay: A comparison of Landsat MSS to TM imagery. Remote Sens. Environ. 22, 235248. https://doi.org/10.1016/0034-4257(87)90060-5

Briggs, I.C., 1974. Machine Contouring Using Minimum $\begin{array}{llr}\text { Curvature. } & \text { Geophysics } & 39,\end{array}$ https://doi.org/10.1190/1.1440410

Campbell, J.B., Wynne, R.H., 2011. Introduction to Remote Sensing, Fifth Edit. ed. The Guilford Press, New York.

Chayhard, S., Manthachitra, V., Nualchawee, K., Buranapratheprat, A., 2018. Kraben Bay (KKB). Int. J. Agric. Technol. 14, 161-170.
Devlin, M., Bricker, S., Painting, S., 2011. Comparison of five methods for assessing impacts of nutrient enrichment using estuarine case studies. Biogeochemistry 106, 177-205. https://doi.org/10.1007/s10533-011-9588-9

ESRI, 2016. ArcGIS for Desktop [WWW Document]. Environ. Syst. Res. Institute, Inc. URL desktop.arcgis.com/en/arcmap/10.3/tools/3d-analysttoolbox/how-spline-with-barriers-works.htm\#GUID-91458C2F1FFB-4643-BBD4-045D22BDD1D5 (accessed 1.28.20).

Flynn, K.F., Chapra, S.C., 2014. Remote sensing of submerged aquatic vegetation in a shallow non-turbid river using an unmanned aerial vehicle. Remote Sens. 6, 12815-12836. https://doi.org/10.3390/rs61212815

Green, E.P., Short, F.T., 2004. World Atlas of Seagrasses, University of California Press. University of California Press, Berkeley. https://doi.org/10.5860/choice.41-3160

Jensen, J.R., 2014. Remote Sensing of the Environment: An Earth Resource Perspective, Second Edi. ed. Pearson Education Limited, Harlow England.

Koch, E.W., 2001. Beyond Light: Physical, Geological, and Geochemical Parameters as Possible Submersed Aquatic Vegetation Habitat Requirements. Estuaries 24, 1-17.

Kolanjinathan, : K, Kolanjinathan, K., Ganesh, P., Saranraj, P., 2014. Pharmacological Importance of Seaweeds: A Review. World J Fish Mar Sci 6, 1-15 https://doi.org/10.5829/idosi.wjfms.2014.06.01.76195

Komatsu, T., Igarashi, C., Tatsukawa, K., Nakaoka, M., Hiraishi, T., Taira, A., 2002. Mapping of seagrass and seaweed methods beds using hydro-acoustic methods. Fish. Sci. 68, 580-583. https://doi.org/10.2331/fishsci.68.sup1_580

McCarthy, E.M., Sabol, B., 2000. Acoustic characterization of submerged aquatic vegetation: Military and environmental monitoring applications. Ocean. Conf. Rec. 3, 1957-1961. https://doi.org/10.1109/OCEANS.2000.882226

Orth, R.J., Moore, K.A., 1984. Distribution and abundance of submerged aquatic vegetation in Chesapeake Bay: An historical perspective. $\quad$ Estuaries 7, 531-540. https://doi.org/10.2307/1352058

Philippine Statistic Authority, 2015. Census of Population [WWW Document].

Seagrass-Watch, 2010. Seagrass-Watch Monitoring.

Smara, S., Bouchon, C., Maniere, R., 1998. Remote sensing techniques adapted to high resolution mapping of tropical coastal marine ecosystems (coral reefs, seagrass beds and mangrove). Int. J. Remote Sens. 19, 3625-3639. https://doi.org/10.1080/014311698213858

Taddia, Y., Russo, P., Lovo, S., Pellegrinelli, A., 2019. Multispectral UAV monitoring of submerged seaweed in shallow water. Appl. Geomatics 12. https://doi.org/10.1007/s12518-01900270-x

Yuan, L., Zhang, L.Q., 2008. Mapping large-scale distribution of submerged aquatic vegetation coverage using remote sensing. Ecol. Inform. 3, 245-251. https://doi.org/10.1016/j.ecoinf.2008.01.004 